\title{
Is it Still True that Women Live Longer than Men, But with a Worse Quality of Life?
}

\author{
Modena MG* and Martinotti V \\ University of Modena and Reggio Emilia, Italy
}

*Corresponding author: Maria Grazia Modena, University of Modena and Reggio

Emilia, Via del Pozzo 7141121 Modena, IT, Italy, Tel: 059-4224241-4878; Email:

mariagrazia.modena@unimore.it

\author{
Editorial \\ Volume 2 Issue 2
}

Received Date: June 14, 2018

Published Date: June 22, 2018

DOI: $10.23880 /$ whsj-16000116

\section{Editorial}

In 1998 the WHO published a "gender challenge" for national and international organizations, an invitation to better assess risk factors involving women's health, to develop preventive strategies to reduce the impact of more afflicting diseases for women, and a major effort in understanding why men die before women [1]. Among such afflicting diseases, cardiovascular, depression and comorbidities are the most important.

Cardiovascular disease (CVD), in fact, is the leading cause of mortality in Western populations. It is also known that the life-long risk for CVD is higher for men than women. In the last few decades this difference has somewhat narrowed due to a decrease in male, and an increase in female risk, mostly due to a worse lifestyle. Age is the most significant determinant for CVD in both sexes, but the data are not uniform as regards the sex differences in CVD risk by age. A large body of epidemiological evidence supports the concept that in postmenopausal years the female risk for CVD elevates more rapidly than among same-aged men while equally strong data dispute this difference. Women have also been found to have a longer life expectancy in populations with manifest atherosclerosis. These contradicting results may be partly explained by methodological differences, e.g. in statistical analyses used [2]. Anyway, in our opinion there is some protection against atherosclerosis in women, because independently on these different observations, it is evident that women still live more than men, although with more comorbidities. We would like to discuss why women live longer and why with a worse quality of life. Women are less likely to receive preventive treatment or guidance, such as lipid-lowering therapy, aspirin and therapeutic lifestyle changes than men at similar risk [3]. When medications are prescribed, treatment is less likely to be aggressive or to achieve optimal effects, for example, women with hypertension are less likely to have their blood pressure at goal, and hyperlipidemic women, especially those with coexisting diabetes mellitus, are less likely to be treated with statins to lower low-density cholesterol [4]. In other words, although women are less treated for traditional CVD risk factors they die later for CVD. Again, also when women present severe heart failure, prognosis is better than in men and they are transplanted later in life $[5,6]$. Loss of ovarian hormones either naturally or surgically increases the aging processes in women, including incidence, morbidity, and mortality from cardiovascular disease. Observational studies, which evaluated associations among endogenous levels of the sex steroids with CV risk and event incidence in postmenopausal women, mostly indicate that risk and incidence increase with decreases in endogenous estrogen. I will not discuss the issue of hormonal replacement therapy, now redefined menopausal hormonal therapy, but the fact that new trials are ongoing means that the role of estrogen on the cardiovascular system is still a fascinating and unclear subject. In our opinion a kind of "estrogenic memory" influence and protect women's life [7].

On the other hand, in fact, new emerging, nontraditional atherosclerotic risk factors, include preterm delivery, hypertensive pregnancy disorders, gestational diabetes mellitus, breast cancer treatments, autoimmune diseases, and depression and all are somewhat related to a disorder of hormonal female 


\section{Women's Health Science Journal}

status. Some of these risk factors (i.e. depression) and comorbidities (autoimmune disease) are the main reason of a worse quality of life in female elderly population.

Moreover, there are other nonscientific considerations that give the reason of a worse quality of life in our society for females. If Plato and Cicero in their works diffused a not entirely negative idea of seniority, reevaluating the wisdom, the background, the dissociation from physical desires and the consequent major willingness towards meditation of elderly people (referring almost exclusively to the male gender), in later times the condemnation of seniority became more explicit and generalized. Negative traits characterizing senescence are obviously the expulsion from the labor market, the physical and mental decay, the reduced elasticity of thought, the reduced sexual activity, the solitude, the celebration of the past, the conservatism. Especially today, in the "orgy of juvenileism" that invades economy, medicine, advertising, leisure and entertainment, becoming old is considered as a problem, even a fault, something to hide: especially if you are a woman. The old woman (we usually do congratulate a handsome old man, never a beautiful old woman!) is mocked, portrayed in art as repellent, vicious, malicious, unclean, untidy: a sort of a witch, basically, referring to the funereal and cruel image of death. She is saved only when she is restricted in a maternal and protective role, if she takes care of her grandchildren and tells them fairy tales: in fact, during physical decay (falling of teeth and hair, dry skin, weakening of limbs, loss of memory) only the voice can be saved, dedicated to the narration, to the singing, to the comforting words. The ghettoization of the woman in menopausal age derives from the excessive consideration always given to maternity, the only universally recognized value to women: when fertility and reproductive capacity are lacking, the sterile condition becomes synonymous with social and sexual undesirability and uselessness. There is also a sort of derision or discomfort, even if not emphasized as in the past, for the woman agée who claims her right to love and sexuality, which is not denied to the male, who keeps intact his procreative qualities and his right to paternity even at a very advanced age. For example, I am convinced, even if I never cheered for her, that if Hillary Clinton lost against Barack Obama, and then again against Donald Trump (sic), it is because the former had the charm of the young man and the second had the charm of the old man (and money), against whom the old woman cannot compete.

Experiments are needed to better understand the complex hormonal environment affecting cellular and organ functions involved in development and progression of $\mathrm{CV}$ disease in women as they age.

\section{References}

1. WHO (1998) The World Health Report 1998, Geneva.

2. Mikkola TS, Gissler M, Merikukka M, Tuomikoski $\mathrm{P}$, Ylikorkala $O$ (2013) Sex differences in age-related cardiovascular mortality. PLoS One. 8(5): e63347.

3. Garcia M, Mulvagh SL, Bairey Merz CN, Buring JE, Manson JE (2016) Cardiovascular Disease in Women Clinical Perspectives. Circ Res 118(8): 1273-1293.

4. Lodi E, Carollo A, Martinotti V, Modena MG (2018) Hypertension and Pharmacological Therapy in Women. High Blood Press Cardiovasc Prev 25(2): 147-150.

5. Grigioni F, Barbieri A, Russo A, Reggianini L, Bonatti S, et al. (2006) Prognostic stratification of women with chronic heart failure referred for heart transplantation: relevance of gender as compared with gender-related characteristics. J of Heart and Lung transplant 25(6): 648-652.

6. Bozkurt B, Khalaf S (2017) Heart failure in women. Methodist Debakey Cardiovasc J 13(4): 216-223.

7. Modena MG (2016) Estrogens and the Heart: Do they Help or Hurt? How Estrogen impacts the Cardiovascular System. SOJ Gynecol Obstet Womens Health 2(1): 8.

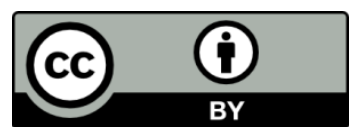

Modena MG and Martinotti V. Is it Still True that Women Live Longer than Men, But with a Worse Quality of Life?. Womens Health Sci J 2018, 2(2): 000116. 